\title{
Téoros
}

Revue de recherche en tourisme

\section{Offre et demande} Un pont à consolider

\section{Marie-Andrée Delisle}

Volume 17, numéro 2, été 1998

L'industrie touristique autochtone

URI : https://id.erudit.org/iderudit/1072267ar

DOI : https://doi.org/10.7202/1072267ar

Aller au sommaire du numéro

Éditeur(s)

Université du Québec à Montréal

ISSN

0712-8657 (imprimé)

1923-2705 (numérique)

Découvrir la revue

\section{Citer cet article}

Delisle, M.-A. (1998). Offre et demande : un pont à consolider. Téoros, 17(2),

5-12. https://doi.org/10.7202/1072267ar

Ce document est protégé par la loi sur le droit d'auteur. L'utilisation des services d'Érudit (y compris la reproduction) est assujettie à sa politique d'utilisation que vous pouvez consulter en ligne.

https://apropos.erudit.org/fr/usagers/politique-dutilisation/
Cet article est diffusé et préservé par Érudit.

Érudit est un consortium interuniversitaire sans but lucratif composé de l'Université de Montréal, l'Université Laval et l'Université du Québec à Montréal. Il a pour mission la promotion et la valorisation de la recherche. https://www.erudit.org/fr/ 


\title{
Offre et demande
}

\author{
UN PONT À CONSOLIDER
}

\section{Marie-Andrée Delisle}

Évaluer le potentiel du marché et les opportunités en matiere de tourisme autochtone est relativement facile, puisque son positionnement se renforce de jour en jour et que l'intérêt pour cette riche culture croît de façon exponentielle. Or, lorsqu' on y regarde de près, on se rend compte qu'il est très difficile d'évaluer la portée des opportunités de marché, sans parler de la qualité de l'offre actuelle. End'autres mots, les opportunités sont présentes, mais leur concrétisation dépend largement de la qualité et de la fiabilité des prestations' touristiques autochtones que l'on propose. Le présent article vise donc à cerner les opportunités et le potentiel de certains marchés, tout en situant dans leur ensemble certains facteurs déterminants.

A la suite d'entrevues, d'observations sur le terrain, de recherches, de participation à divers salons professionnels, ainsi que d'échanges avec des acheteurs professionnels, nous dresserons un portrait des grandes opportunités de marchés que propose le tourisme autochtone, tout en soulignant les attentes et les exigences de ces marchés.

Nous verrons les eléments sur lesquels reposent les opportunités de marché en matière de tourisme autochtone et les marchés porteurs auxquels devraient principalement être affectés les budgets de marketing.

\section{UN TOURISME AUTOCHTONE DÉJÀ BIEN EN PLACE}

Le tourisme autochtone est plus qu'en devenir, il est présent partout ! En plus des sites déjà rondement exploités (comme le Site Onhoüa Chetek8e de Wendake et le Musée amérindien de Pointe-Bleue), dans la plupart des régions du Québec de nombreux autres sites sont soit en développement, soit en voie d'ouvrir leurs portes. Mieux connu parce que davantage médiatisé et positionné, le tourisme autochtone devient peu à peu incontournable dans les itinéraires que l'on propose aux Européens, qu'ils voyagent en groupe ou de façon individuelle. En fait, l'Europe, principalement la France et l'Allemagne, en redemande. Depuis l'événement d'Harricana ${ }^{2}$, où la couverture médiatique a valu plusieurs millions en dollars publicitaires, la France - qui rêve encore
d'Indiens aux plumes colorées, brandissant le tomahawk devant leur tipi familial, leur authentique résidence - a découvert le style de vie traditionnel des Autochtones en forêt. Quant aux Allemands, ils rêvent toujours des Indiens du Far-West et de leurs épopées avec les cowboys américains ; en contrepartie, tout comme les Français, ils sont devenus très sensibles aux revendications des Autochtones tant en ce qui concerne le maintien de leurs traditions que leurs territoires ancestraux. La demande étant vive et pressante, l'offre autochtone se trouve dans une situation stratégiquement intéressante, mais fragile, puisque les prestations ne sont pas encore totalement arrimées aux exigences de l'industrie.

Les Autochtones veulent partager leurs valeurs et leurs traditions, de façon simple et conviviale. Très réservés de nature, ceux qui proposent des expériences de courts séjours en mode de vie traditionnelle ont plutôt tendance à répondre aux questions au fur et à mesure qu'elles sont soulevées, sans avoir nécessairement structuré leur approche communicationnelle et sans avoir intégré d'animation dans leurs discours. Pourtant, la richesse de leur vécu et des traditions qui s'y rattachent donne à leurs propos une valeur ajoutée incontestable. En contrepartie, le touriste s'attend à vivre une expérience romancée qu'il ne trouvera pas forcément lors de son passage chez les Indiens, à moins qu'il ne soit bien préparé à l'expérience qui l'attend. En effet, si les Autochtones se rendent toujours sur leurs lignes de trappe familiales, ils habitent néanmoins des villages et des quartiers nord-américains, dans des maisons où le confort et la modernité font partie de leur quotidien. Les touristes sont donc parfois étonnés de ne pas retrouver intactes les images des magazines de voyages qui les ont amenés chez les Indiens. Pour être viable, l'offre touristique autochtone doit donc être correctement présentée et vendue, dans un continuum plutôt que dans une approche disneyesque. Si le costume aide parfois à créer un personnage, il constitue en même temps un couteau à deux tranchants puisqu'il perpétue l'image de l'Indien à plumes.

\section{UN INVENTAIRE EN MOUVEMENT}

Si l'on évalue les différentes prestations touristiques présentement sur le marché, on remarque deux grands types d'offre. D'abord, l'offre qui peut être proposée au tourisme de masse, par exemple, le 
Musée amérindien de Pointe-Bleue (Mashteuiatsh) au Lac-Saint-Jean, la Maison de transmission de la culture à SeptIles, le site reconstitué Site Onhoula Chetek8e du Village huron de Wendake en banlieue de Québec, ou le Fort Listuguj de Restigouche en Gaspésie, dont les caractéristiques principales sont la présentation d'un site reconstitue ou d'un site d'interprétation culturelle ainsi que la proximité soit d'un axe routier qu'empruntent les circuits d'autocars, soit d'un centre urbain où passent ces circuits. Ce sont des sites qui sensibilisent à des modes de vie et à des thématiques particulières ; leur contenu et leur animation sont généralement bien structurés puisqu'ils doivent s'insérer dans les horaires assez chargés des clientèles d'autocars.

L'autre type d'offre concerne davantage l'axe culture-nature-aventure, comprenant des propositions d'expériences en territoire avec séjour chez les Autochtones : ce sont les petits groupes et les voyageurs individuels qui sont particulièrement intéressés à ce type d'expérience qui laisse davantage place à un échange de cultures, à l'apprentissage d'autres valeurs et à l'expérimentation d'un mode de vie différent. L'Européen voudra bien vivre la vie d'un Amérindien pendant quelques jours - mais pas vraiment plus _ , dans un contexte d'authenticité et de participation, dans le but de vivre une expérience unique.

L'offre autochtone actuelle comprend plusieurs produits destinés au tourisme d'autocar, avec des sites relativement bien fréquentés (Site Onhoüa Chetek8e à Wendake : plus de 100000 visiteurs par année; Musée amérindien de PointeBleue : environ 35000 visiteurs par année ; Fort Listuguj : 14000 visiteurs pour une première année d'activités et plus de 1000 pour le village cri d'OujeBougoumou, situé à 57 kilomètres de Chibougamau, pour l'été 1997. En contrepartie, l'expérience en territoire autochtone est beaucoup plus restreinte en matière de capacité d'accueil : les entreprises qui offrent des séjours ethno-culturels en mode de vie traditionnelle ne peuvent recevoir que quelques dizaines de personnes à la fois, tant dans un souci de conserver l'authenticité que de bien livrer la prestation. Une vérification sommaire nous amène à évaluer la fréquentation à quelques dizaines de groupes par année (Aventure Mikuan II près de Roberval,

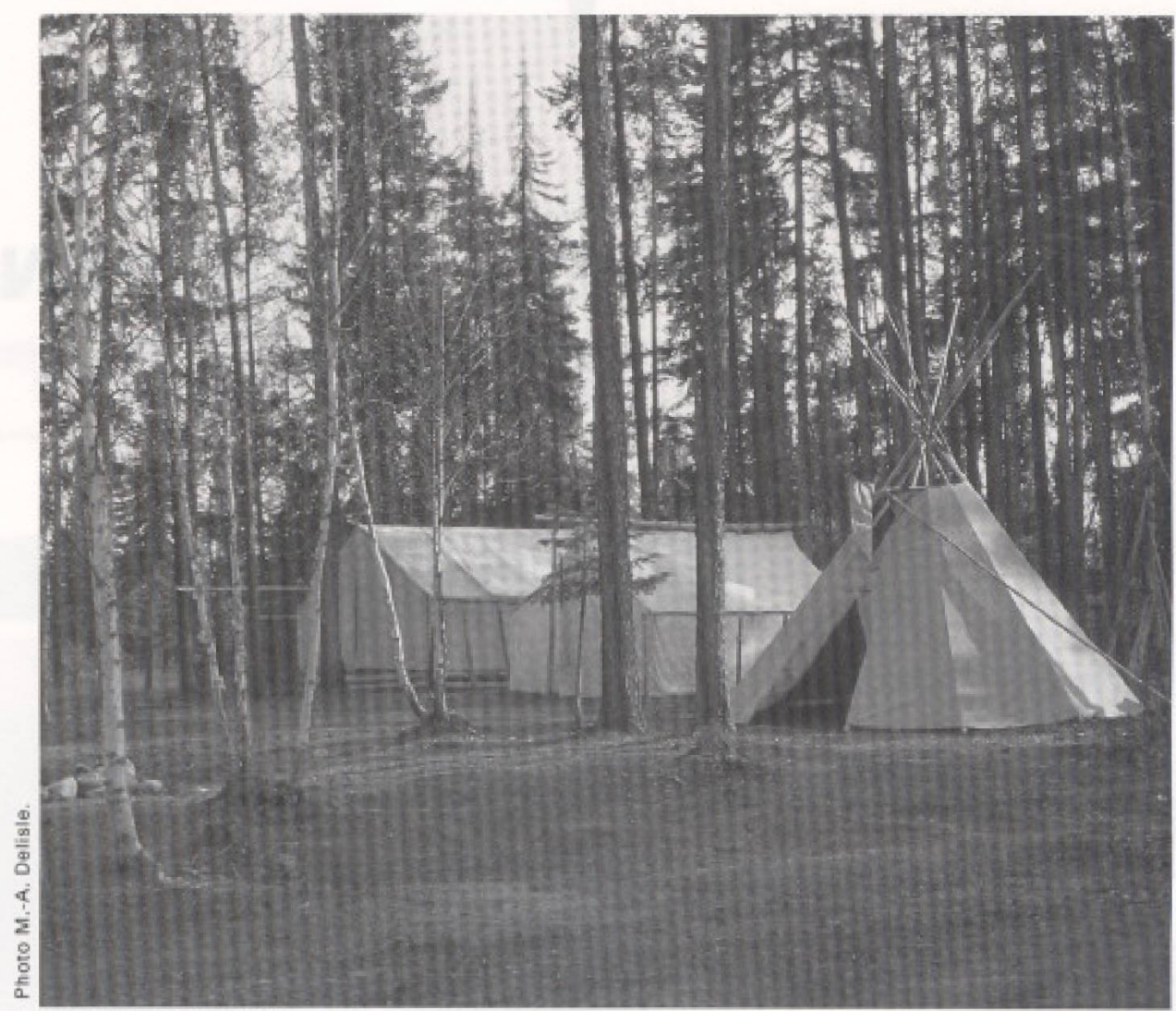

Aventure Mikuan II.

Nessipi Kantuet près de Schefferville, Cultural Tours à Ouje-Bougoumou), tout au plus à quelques centaines de personnes par année. Rappelons que ces sites sont parfois assez éloignés des grands centres et que cela peut devenir un handicap pour les touristes qui ne séjournent pas assez longtemps au Québec et qui, en conséquence, manquent de temps pour une incursion en territoire autochtone. Une telle expérience cadre aussi bien sous le vocable de tourisme d'aventure douce, de tourisme ethno-culturel ou d'écotourisme, ce qui permet d'élargir l'éventail des clientèles possibles.

S'il existe effectivement une trentaine de sites touristiques autochtones (sans compter les boutiques d'artisanat et les festivals), on remarque par ailleurs une certaine répétition dans l'offre: les sites d'interprétation sont nombreux, mais ne proposent pas nécessairement de distinctions autres que la thématique. De son côté, l'expérience en territoire se solde fréquemment par le même type de forfait : coucher sous le tipi, interprétation de la culture, échange avec les aînés, dégustation de mets traditionnels. C'est donc, d'une certaine façon, une thématique particulière qui aide le visiteur à choisir un site plutôt qu'un autre et à l'intégrer à son itinéraire.

En plus des différences régionales, il y aurait done lieu de faire ressortir les distinctions culturelles des différentes nations, de sorte que chaque expérience devienne unique. Cela peut sembler exigeant, mais les opportunités de marché sont bien présentes et il serait dommage de diluer l'offre en oubliant de faire intensément ressortir les particularités culturelles, historiques et environnementales de chaque site.

\section{LE PARTENARIAT AVEC DES RÉSEAUX EXISTANTS}

Toujours en ce qui a trait aux opportunités de marché, l'offre autochtone doit nécessairement s'insérer dans des circuits existants. Les promoteurs et les fournisseurs de produits et services touristiques autochtones ont tout intếrêt à travailler en réseau avec les responsables d'autres attraits régionaux, afin d'attirer le plus grand nombre possible de touristes et de bénéficier de retombées économiques importantes. Cela ne signifie pas pour autant que l'authenticité soit sacrifiéc. À 
titre d'exemple, si l'on planifie un premier voyage en France, on tentera de voir plusieurs aspects du pays plutôt que de s'en tenir à une thématique, comme les vignobles ou les musées. Il en va de même pour les Européens qui, ne l'oublions pas, viennent d'abord visiter le Canada en tant que destination et couvrent généralement deux provinces, par exemple le Québec et l'Ontario.

Les organismes de promotion tels que la Commission canadienne du tourisme, Tourisme Québec, les associations touristiques régionales ainsi que les offices de tourisme locaux, représentent tous des opportunites de développement de marché intéressantes. Par exemple, Chibougamau inaugurait son nouveau Centre minier l'été dernier, au même moment où le village voisin d'Ouje-Bougoumou inaugurait ses visites du village, ce qui permettait de miser sur deux efforts de promotion à la fois, l'un allochtone et l'autre autochtone. Conjointement, ces efforts ont renforcé le positionnement de la Route du nord qui relie la région du Lac-Saint-Jean à celle de la Baic-James. On offrait ainsi aux visiteurs de voir un maximum d'attraits, ce qui constituait pour eux autant de raisons de faire le détour.

\section{LES ATOUTS ET LES FRAGILITÉS}

Si l'on traite d'opportunités, on doit tout autant considérer les atouts et les fragilités de l'offre touristique autochtone. La volonté de faire connaître leur culture et de bien recevoir leurs visiteurs, le désir de bien faire et le goût de développer des projets touristiques, sont autant d'atouts. que détiennent les Autochtones. La fragilité vient plutôt de la méconnaissance des rouages et des exigences de l'industrie, bien compréhensible étant donné le peu d'expérience en tourisme de la plupart des intervenants autochtones. Le manque de formation en gestion et les difficultés de financement, la difficulté d'orchestrer la promotion et la distribution du produit sont autant d'obstacles pour l'entrepreneur autochtone.

Afin de pallier cette situation, la Société touristique Innu (STI) ${ }^{3}$ propose de relever le défi avec détermination ; ses membres doivent respecter certaines normes de gestion, d'encadrement et de service, $s$ 'ils veulent faire partie du plan de com- mercialisation national et international de la STI (voir autre article dans la présente publication). Le fait d'établir des normes, compatibles avec celles généralement exigées par l'industrie, permet alors aux entreprises autochtones de faire du * réseautage $\$$ et de bâtir leur crédibilité face aux acheteurs possibles, tels que les agences réceptives du Québec et les voyagistes européens.

\section{LE DÉVELOPPEMENT TOURISTIQUE ET LA COMMUNAUTÉ}

Si les opportunités de marché sont réelles, elles doivent tout de même être développées avec l'accord des communautés en cause. Or, certaines traditions qui font partie intrinsèque du mode de vie des Autochtones, notamment partir à la chasse à une période précise de l'année, ou encore offrir ses respects lors du décès d'un aîné, empêchent parfois de livrer l'activité pour laquelle on s'était engagé. Voici quelques exemples des questions que doivent se poser les communautés avant de répondre aux opportunités de marché : Quelle sera la ligne de démarcation entre la protection des valeurs et des traditions qui ne regardent que la communauté et les exigences précises des promesses faites au client ? Jusqu'où l'offre peut-elle être développée sans ébranler les traditions ? Jusqu'à quel point peut-on consentir au désir un peu voyeur des touristes qui veulent toujours repousser les limites des secrets ?

\section{L'IMPORTANCE DE LIER LA PROMOTION À LA DISTRIBUTION}

La Commission canadienne du tourisme (CCT) a traduit sa volonté d'aider les entreprises touristiques autochtones par divers efforts de promotion, principalement sur le marché européen, en facilitant la présence des fournisseurs autochtones lors des bourses touristiques à Londres et à Berlin. Le document intitulé Éveillez vos esprits, Guide de l'expérience autochtone au Canada, publié en 1997, propose certaines activités selon les différentes régions du Canada, sans toutefois donner, à notre avis, suffisamment de détails sur les prestations de chacun des fournisseurs. Sa distribution aura cependant intéressé nombre d'acheteur's professionnels qui cherchent depuis à concrétiser des propositions d'activités avec des fournisseurs autochtones. Dans la foulée du défunt comité marketing qu'elle avait spécifiquement mis sur pied pour le tourisme aborigène, la CCT intègre maintenant ses efforts de marketing dans le cadre de ses autres activités, selon les marchés visés. Elle a effectué certaines études de marché et continue de collaborer étroitement avec I'Aboriginal Tourism Team Canada, organisme qui regroupe les intérêts des divers acteurs de l'industrie en matière de tourisme autochtone. Pour la période 19981999 , la CCT a affecté un budget de 800000 \$ à son programme d'activités visant le tourisme autochtone en Europe ; cela comprend entre autres des missions dans diverses villes européennes, ainsi que la participation au World Travel Market de Londres et l'International Tourist Bourse (ITB) de Berlin. Enfin, la CCT a maintenu son comité marketing pour le tourisme d'aventure et considere que le tourisme autochtone devrait stratégiquement s'intégrer à cette thématique.

\section{LES AVANTAGES DU RÉSEAU DE DISTRIBUTION}

Plusieurs entreprises autochtones sont fières d'avoir déjà créé des liens avec des acheteurs européens. Cependant, elles doivent évaluer le coût de leur présence aux événements professionnels ainsi que leur niveau d'efficience, car si l'engouement des acheteurs est là, la vente fait souvent défaut, pour toutes sortes de raisons : manque d'information concrète, claire et précise, itinéraires mal structurés, erreurs dans les tarifs, retards à répondre aux demandes, etc.

Les démarches en direct amènent des risques tant pour le fournisseur que pour le produit en général. C'est pourquoi Tours Innu, nouveau réceptif autochtone au Québec, propose une alternative intéressante, celle de représenter plusieurs nations et plusieurs produits tout en assurant une qualité de prestation essentielle au succès du tourisme autochtone.

\section{LES CLIENTËLES POTENTIELLES}

L'engouement des Français et l'intérêt des Allemands pour le fait autochtone en font des clientèles prioritaires. Si les grands 
salons de Paris, de Londres et de Berlin représentent des débouchés de premiêre importance, ils le sont à la condition d'arrimer les images folkloriques à l'offre touristique actuelle ; à la condition aussi de faire valoir les trois sous-thèmes du tourisme aborigène, soit la culture de ses nations, la grande nature dans laquelle se trouvent ses sites ancestraux et l'aventure dans un mode de vie différent et unique.

Le potentiel de voyageurs individuels nord-américains se trouve plutôt du côté de la motoneige, de la chasse et de la pêche. Pourtant le marché de groupes nord-américains est certes propice aux attraits autochtones structurés pour recevoir du tourisme de masse. Outre certaines foires américaines, des salons québécois permettent de solliciter les deux types de clientèles : Salon du Grand Air, Salon Vacances et Loisirs d'été, Salon de la pourvoirie, Exposition touristique et Bourse Bienvenue Québec.

\section{UNE AUTRE AVENUE : LE MARCHÉ DE L'ÉCOTOURISME}

De nombreuses études de marché confirment que l'écotourisme croît à une vitesse phénoménale ; pourtant, ce segment de marché - qui s'adresse non seulement aux touristes soucieux de respecter l'environnement, mais aussi à ceux qui veulent observer la nature dans des conditions d'aventure douce et de confort, ou connaître d'autres cultures en recherchant une expérience humaine enrichissante plutôt que le luxe - demeure mince. Un tel marché intéresse principalement des individus, à la grandeur de la planète, et englobe invariablement la notion de développement durable. Il existe deux principales avenues pour le connaître: les voyagistes spécialisés en tourisme d'aventure ou en écotourisme et l'Internet. Il faut cependant être conscient des exigences très strictes de ce segment de marché, tant en matière de protection du patrimoine humain et naturel que de développement durable dans les vitrines proposées.

\section{LES ATTENTES DES FRANCAIS ET DES ALLEMANDS}

A la suite de différentes recherches effectuées par la CCT et par la direction du

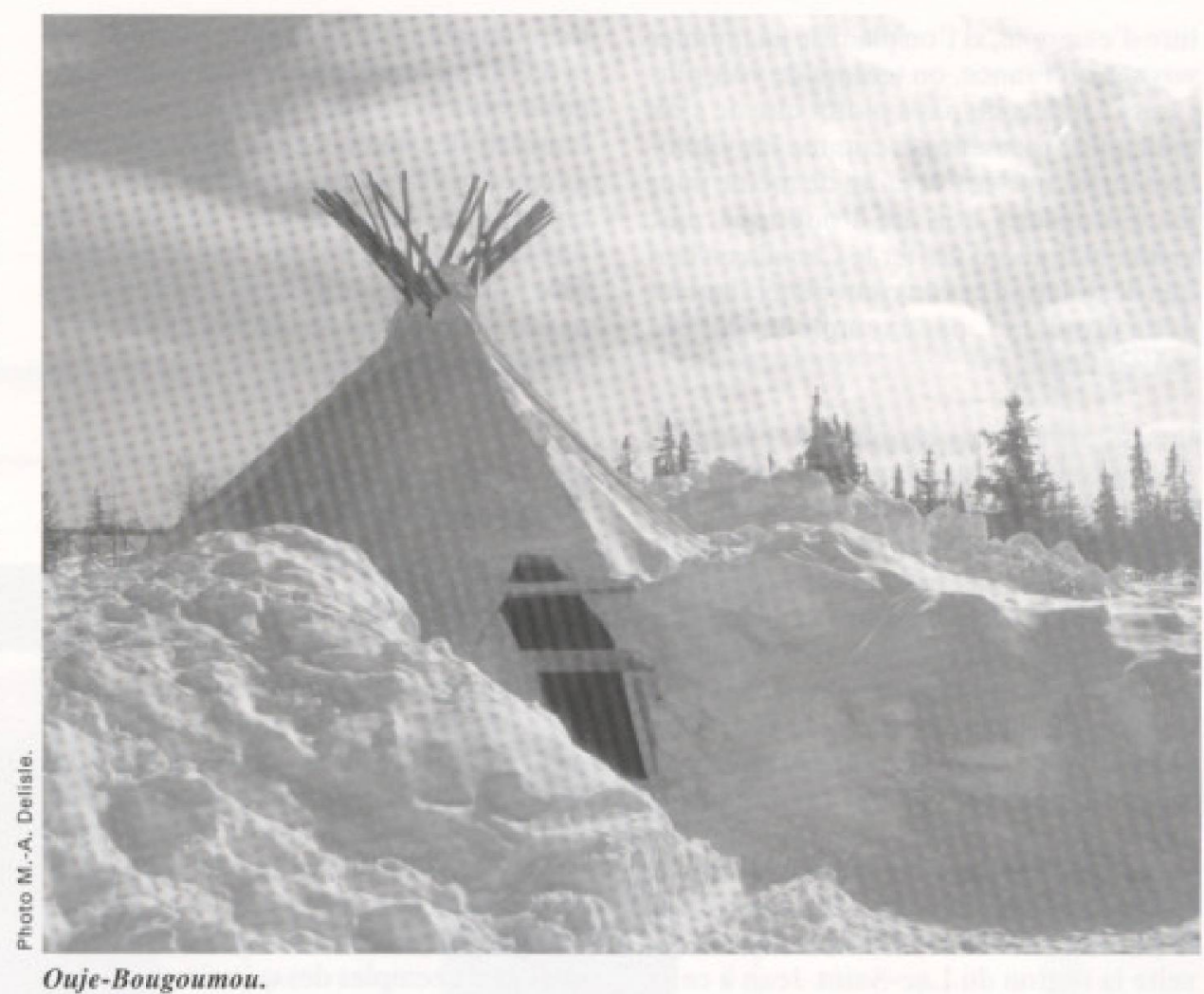

développement des marchés de Tourisme Québec, il appert que les marchés français et allemand sont les deux clientèles qui offrent le plus de potentiel de développement (voir encadré sur les marchés potentiels). Parmi l'équipe de démarchage de Tourisme Québec qui auvre sur le continent curopéen, Martina KlöcknerScherfeld et Barbara di Stefano s'occupent respectivement des marchés allemands et français. Lors de son passage à Rendez-vous Canada à Québec en mai 1998, Martina Klöckner-Scherfeld soulignait que bien que l'Allemagne soit considérée comme un marché prioritaire pour le produit autochtone au Québec, les Allemands n'en connaissent pas grand chose : * Ils se font une image romantique des Indiens et s'attendent à quelque chose de folklorique *. Évidemment, ils sont déçus lorsqu'ils aperçoivent les villages autochtones qui ressemblent à peu de choses près à d'autres villages du Québec, puisqu'ils entretiennent encore une image Far-West des habits et des modes de vie amérindiens.

En fait, ils veulent voir quelque chose de different, quelque chose qu'on ne peut retrouver nulle part ailleurs. Ils veulent savoir comment vivent les autochtones aujourd'hui, sans pour autant en savoir suffisam. ment avant de partir, ce qui pourrait nuancer leurs attentes. D'ailleurs, ils circulent principalement sur les axes couvrant l'Ontario et le Québec et plus précisément Montréal - Québec - Tadoussac et Gaspésie, souvent en maison motorisée ou en voiture de location. Ils recherchent davaniage une courte incursion chez les Autochtones, une expérience hors des sentiers battus, en autant que ce n"est pas trop loin de leur axe principal.

Selon la déléguée allemande de Tourisme Québec, les prestations doivent offrir de meilleures garanties de service : elles doivent être mieux définies en terme de contenu, de durée et de qualité de livraison ; on doit mieux expliquer le mode de vie, faire des randonnées d'interprétation avec une bonne animation, mettre en contexte les éléments des plats traditionnels. Le produit demande à être peaufiné s'il veut correspondre aux normes du marché international ! $\%$, ajoute-t-elle. Lorsque le guide ne se présente pas, lorsque l'activité est annulée, lorsque le contenu est mince, lorsque la tente est délabrée, le produit devient dangereux à vendre surtout en Allemagne où les lois sur la protection du consommateur sont strictes; le touriste allemand prendra recours 
et sera dédommagé pour ce qu'il n’a pas vu ou expérimenté ainsi que pour perte de jouissance.

Toujours selon Martina KlöcknerScherfeld, les Allemands n'hésiteront pas à faire cing heures de route pour voir quelque chose d'authentique ; ils privilégient le village en territoire et l'expérience en pleine nature les intéresse plus que le centre d'interprétation ou le site reconstitué, lesquels devraient compléter l'expérience sur le terrain. En outre, il est essentiel, pour la clientèle allemande, d'avoir à tout le moins un guide qui peut s'exprimer en anglais, Elle souligne aussi l'importance d'offrir des sacs de couchage propres, d'assurer un confort minimum avec matelas, de garder la tente au chaud, d'inclure des activités sportives telles que le canot, la randonnée en raquettes, en ski de fond ou à pied. Au plan de la nourriture, les mets traditionnels seront prisés en autant qu'ils soient authentiques : $*$ Les Allemands ne sont pas gourmands, ils mangent pour se nourrir! $\%$, selon $\mathrm{M}^{\mathrm{me}}$ Klöckner. Pour mieux vendre le produit, il faudra donc recréer un environnement authentique coherent et bien camper les liens historiques, culturels et traditionnels du passé, du présent et de l'avenir. Le $T$-shint bleu ciel ou les $\alpha$ godasses $\$$ Nike sous le costume traditionnel, les mets traditionnels servis dans des assiettes de plastique, le site de campement à deux kilomètres de la route (le bruit le rappelle la nuit), sont autant d'éléments que remarqueront les visiteurs européens en manque d'authenticité !

Pour ce qui est des Français, Barbara di Stefano souligne que le fait autochtone les intéresse grandement et recèle toujours un petit quelque chose de magique qui les fait parfois tomber dans le folklore. Ils gardent souvent l'image de l'Indien à plumes et c'est ce personnage qu'ils veulent rencontrer afin de se mettre en phase avec lui, mais ils ne soupçonnent pas ce qu'une telle attente peut représenter. Or, le fait que certains Autochtones perpétuent l'image d'Indiens portant des plumes finit par fausser les pistes. Bien qu'ils se documentent largement avant le départ, les Français ne connaissent pas l'histoire des Autochtones au Québec. Ils ne peuvent donc imaginer le contexte actuel des différentes nations et de leurs divers environnements.

Selon $\mathrm{M}^{\mathrm{m}}$ di Stefano, le Français qui vient au Québec en voyageur individuel cher- che à expérimenter la vraie vie des autochtones en forêt, mais en courts séjours. Il sera intéressé à goûter les mets traditionnels et à dormir sous la tente pour une nuit ou deux, pas plus. Il préférera vivre comme un Amérindien pendant deux jours, en autant que l'expérience et l'environnement soient authentiques et typiques, plutôt que de visiter des reconstitutions de sites, trop adaptées à son goût.

En France, Tourisme Québec priorise le marché du voyageur individuel plutôt que le marché de groupe où l'intérềt pour les autochtones demeure trèss folklorique, donc éloigné de la réalité.

En matière de recommandations, $\mathrm{M}^{\mathrm{ms}}$ di Stefano souligne la nécessité de propreté et de confort pour les couchers sous la tente et pour les toilettes sèches. Elle conclut que

Les activités doivent commencer à l'heure, elles doivent être structurées, avoir du contenu. En fait de repas, les Français préféreraient une belle salade composée avec un bout de viande d'ours plutôt que de ne goûter que des viandes de bois en quantité ; mais si on les avise que ces façons de manger sont typiques, alors ils se conformeront joyeusement.

\section{LA PARTICIPATION DIMINUE LE BESOIN D'ENCADREMENT}

Dans le déroulement des activités, on suggère de faire participer les gens et de les mettre à l'épreuve : monter un tipi, allumer un feu, tresser une raquette ou préparer la bannique. Les activités devront toutefois être bien animées par le guide ou l'interprète autochtone, car plus le Français sera passif, plus il aura besoin de structure et d'encadrement dans ses activités. On suggère aussi d'intégrer des activités physiques (canot, marche, trappe, etc.) et de concentrer le contenu sur un, deux ou trois jours, en un même endroit, plutôt que d'étendre les activités en lais= sant trop de temps libre, d'où le sentiment de perte d'occasions que pourra ressentir le touriste européen.

Rappelons que le Français qui voyage individuellement ne saura probablement pas qu'il existe des propositions touristiques concrètes d'expériences de vie tra- ditionnelles avant d'arriver au Québec. II aura lu certains articles de magazines à ce sujet, sans toutefois apporter les coordonnées de ces prestations ; il ira plutôt s'informer, une fois arrivé, dans un centre Infotouriste à Montréal ou à Québec.

\section{CONCLUSION}

Nous avons vu que les opportunités de développement de clientèles pour le tourisme autochtone sont tout à fait réelles et très prometteuses. La promotion, la connaissance des marchés et de leurs exigences, la décision d'une communauté de miser sur le tourisme tout en protégeant ses traditions et ses valeurs, sont autant de facteurs qui lient l'offre à la demande. Les entreprises touristiques autochtones devront investir dans la formation de leur personnel afin d'améliorer la qualité du service à la clientèle qui doit nécessairement s'appuyer sur des normes qui permettent la récurrence et la constance des façons de faire : être accessible et répondre prestement aux demandes, être à l'heure, être présent, être authentique, savoir communiquer, présenter un contenu structuré et animé, etc.

En considérant la capacité d'accueil, l'accessibilité et le degré de complémentarité avec d'autres sites, le gestionnaire de prestation touristique autochtone aura de meilleurs paramètres pour décider vers quels marchés porteront ses efforts et quels types d'acheteurs devront être sollicités. De plus, les réseaux de distribution et de promotion devront savoir bien vendre le produit autochtone : courber l'imaginaire, favoriser des attentes réalistes, souligner que les Autochtones se sont intégrés à la vie courante.

Le tourisme autochtone expose une image forte, qui se doit d'être égale à elle-même, du début à la fin. Plus l'expérience autochtone sera authentique et distinctive, plus vite les communautés profiteront des retombées économiques significatives. Elles seront alors en mesure de mieux profiter des opportunités de marché, tout en s'appuyant sur les stratégies de marketing prévues dans la démarche de promotion de Tourisme Québec et de la Commission canadienne du tourisme. 


\section{LES DÉMARCHES DE TOURISME QUÉBEC POUR METTRE EN VALEUR L'OFFRE AUTOCHTONE}

Depuis quelques années, la direction du développement des marchés de Tourisme Québec travaille à propulser le produit touristique autochtone sur le marché européen. Membre des comités nationaux sur le tourisme aborigène de la Commission canadienne du tourisme et de l'Aboriginal Tourism Team Canada, Tourisme Québec demeure un intervenant proactif à plusieurs égards.

Parmi ses différentes actions, Tourisme Québec a collaboré à la publication du guide Le Québec autochtone $e^{4}$ et contribué au lancement de ce guide lors du salon Rando-Expo à Paris, en 1997. Il a aussi rendu possible la publication du document Le Québec amérindien et inuit ${ }^{5}$, qui a fait l'objet d'une distribution auprès de tous les médias et les voyagistes d'outre-mer, ainsi que d'un lancement lors de la bourse Rendez-vous Canada de 1997 à Vancouver. Profitant de la tenue du dernier Rendez-vous Canada à Québec en mai 1998, Tourisme Québec a remis aux 400 participants de l'extérieur (médias et voyagistes) une pochette sur le produit autochtone, comprenant des renseignements sur diverses entreprises autochtones telles que la Société touristique Innu, Tourisme Ouje-Bougoumou, Tourisme Kahnawake et la Corporation touristique du Nunavik. Une vingtaine de médias et de voyagistes internationaux ont effectué une tournée post-Rendez-vous Canada, organisée par Tourisme Québec et la Société touristique Innu, afin de se familiariser avec des expériences et des sites autochtones. Plusieurs entreprises autochtones (Société touristique Innu, Musée amérindien de Pointe-Bleue, Corporation touristique du Nunavik, Tourisme Ouje-Bougoumou) ont également profité du support de Tourisme Québec afin de participer à des événements professionnels tels que Rando-Expo et Ateliers Canada à Paris.

En matière de marketing. Tourisme Québec travaille étroitement avec la Commission canadienne du tourisme dans le cadre de programmes conjoints pour l'ensemble du produit autochtone.

Par ailleurs, en ce qui a trait à l'intelligence de marché, la direction du développement des marchés a effectué des relevés portant sur le profil des entreprises touristiques autochtones au Québec. Des rencontres avec les agences réceptives du Québec ont également été organisées pour évaluer, avec elles, les meilleurs moyens de commercialiser le produit autochtone et aussi pour identifier les principaux critères nécessaires à son exportation.

La Politique de développement touristique $^{6} \mathrm{du}$ ministre délégué au Tourisme, M. David Cliche, prévoit des activités de développement et de promotion du tourisme autochtone pour 1998-1999, qui s'intégreront

\section{[...] dans une stratégie québécoise de développement de l'écotourisme, la recherche de l'authenticité du produit autochtone et le renforce- ment du professionnalisme dans les services. [...] Tourisme Québec soutiendra le développement par la mise en place d'une table sectorielle sur le tourisme autochtone qui fa- vorisera l'établissement de relations suivies avec l'Association du Nord du Québec et les promoteurs autoch- tones. De plus, à court terme, Tou- risme Québec s'associera avec les producteurs touristiques autochto- nes et le réseau de distribution quebecois pour metre au point un outil de promotion du tourisme autochtone auprès des grossistes européens.}

À ce titre, une récente recherche portant sur l'inventaire des activités et l'analyse des produits significatifs a été effectuée en vue d'identifier les pistes d'intervention stratégique en matière de tourisme autochtone. Lorsqu'elle sera déposée, cette recherche permettra entre autres de prioriser les efforts pour développer l'offre touristique autochtone, de favoriser les types d'entreprises les plus propices à la demande et de circonscrire les activités de promotion les plus pertinentes.

Tel que stipulé dans la Politique du ministre, un document d'appel est présentement en élaboration. Ce document servira d'abord d'outil d'information et de promotion auprès des agences réceptives du Québec et des voyagistes européens, pour ensuite promouvoir les divers aspects touristiques du produit autochtone auprès des touristes internationaux. En outre, un important volet sur la recherche d'intelligence de marché devrait venir compléter les actions de Tourisme Québec en fournissant des données sur les marchés ciblés (profil des visiteurs) ainsi que sur les motifs et les intérêts de ces marchés en matière de tourisme autochtone.

Selon François Goulet, directeur de la Direction développement des marchés à
Tourisme Québec, le tourisme autochtone se situe présentement comme suit :

Les entreprises touristiques autochtones réalisent de plus en plus l'importance d'être bien préparées pour faire face au marche international. L'authenticité des produits doil fortement ressortir puisque, aux yeux des Francais et des Allemands, c'est un élément capital dans l'expérience du produil autochtone. Jusqu'à maintenant, nous avons davantage réagi à des opportunités de marché concermant le tourisme autochtone, mais au cours de cette année nous ferons suite concrètement à ces opportunités en élaborant un plan marketing strucfuré et récurrent, que nous établirons en partenariat avec le milieu autochtone.

Louis B. Parent, Chef du produit aventure et autochtone au sein de la même direction, pour sa part, est confiant que les efforts consentis par les fournisseurs autochtones permettent beaucoup d"espoir. Il faut cependant leur rappeler l'importance de bien structurer leur offre s'ils veulent transiger avec le marché international. Plusieurs sites proposent des expériences en milieu de vie autochtone, mais selon M. Parent, il ne suffit pas de dire « Dites-moi ce que vous voulez et nous allons vous arranger ça! $b$, car on ne peut pas improviser. Il conclut qu'il faut plutôt préciser l'offre, l'encadrer, lui donner un solide contenu culturel et chiffrer la prestation en vue de l'offrir à des intermédiaires de vente. En ce sens, il reste beaucoup de travail à faire.

Par ailleurs, la création de la nouvelle Association touristique régionale (ATR) du Grand Nord permettra aux entreprises autochtones situées dans cette région de se positionner favorablement et stratégiquement, tant au niveau des activités de marketing qu' au niveau du guide touristique qui sera éventuellement publié. L'ATR du Grand Nord représentera trois principaux groupes, soit la nation Crie, la nation Inuit et la région allochtone de la Baie-James, aussi connue sous le nom de Radissonie.

Enfin, tant la direction du développement des marchés que la direction de la promotion coordonnent leurs efforts pour organiser des tournées de presse afin de familiariser les médias et de mieux positionner l'offre autochtone dans sa réalité d'aujourd'hui. 


\section{APERCUU DES POTENTIELS DE MARCHÉ}

Si le tourisme autochtone présente une option particulière et spécifique, il a par ailleurs l' avantage de regrouper plusieurs des motifs de voyage de l'ensemble des touristes dans le monde, soit le désir d'observer la nature, celui d'en connaître davantage sur d'autres styles de vie, celui de découvrir l'histoire et la culture d'autres peuples et enfin celui de vivre une aventure, douce surtout, en tant qu'experience unique. Ceci ctant dit, le potentiel de marché s'élargit considérablement puisque l'un ou l'autre de ces motifs peut être facilement comblé dans l'offre touristique globale d'une destination telle que le Québec. Certains marchés sont très propices au produit touristique autochtone et représentent des opportunités évidentes ; ce sont sans contredit les marchés français et allemand qui remportent la palme.

\section{LE MARCHÉ NORD-AMÉRICAIN}

Si les marchés canadien et américain représentent une partie importante des visiteurs au Québec $(73 \%$ des touristes et $45 \%$ des recettes viennent du Québec, alors que $27 \%$ des touristes et $55 \%$ des recettes proviennent de l'extérieur), nous ne pouvons les considérer comme prioritaires à l'heure actuelle. En effet, malgré que les Américains représentent des recettes évaluées à 775 millions de dollars, comparativement aux Français avec 355 millions et aux Allemands avec 64 millions, ils ne démontrent pas d'intérêt autre que pour les sites répondant aux exigences des voyages en autocar, ou encore les sites proposant des aventures ou des expéditions uniques. C'est peut-être dû au fait qu'ils manquent d'information ou de sollicitation. Du côté canadien et québécois, les couvertures médiatiques des dernières années ont davantage porté sur les revendications des Premières Nations plutôt que de révéler un positionnement à caractère touristique. Ici aussi et sans doute à cause du manque d'information et, en contrepartie, du manque de promotion et de distribution, le produit touristique autochtone est peu connu.

Trois types de marchés nord-américains peuvent cependant être intéressants à solliciter: les voyages en autocar où certains sites près des centres urbains peuvent recevoir de grands groupes; les échanges académiques avec des groupes d'étudiants ou les échanges sur le thème du développement durable ; les adeptes d'aventure sur les sites en territoire autochtone. Il ne faudrait cependant pas abandonner ces marchés au profit de l'Europe uniquement, puisqu'ils représentent tout de même des clientèles très proches, nombreuses et faciles à solliciter.

\section{LE MARCHÉ EUROPÉEN}

Une étude, effectuée pour le compte de la CCT par 1'Université Simon Fraser?, indique que les deux marchés les plus importants à solliciter pour y vendre le produit autochtone sont la France, dont l'intérêt est déjà transformé en décision d'achat, et l'Allemagne, qui démontre un intérêt croissant.

L'étude couvre également le marché italien qui, selon ses voyagistes, semble déjà trouver difficile de vendre le Canada comme destination touristique, donc avec encore moins de possibilités de positionner le produit autochtone dans l'ensemble de la destination. Quant au marché britannique, l'intérêt n'est pas suffisamment développé à l'heure actuelle pour en faire un marché prioritaire. Ces chiffres valent toujours pour l'ensemble du Canada, alors que pour le Québec, les marchés italien et britannique sont en croissance constante depuis trois ans, mais principalement les circuits les plus traditionnels qui couvrent habituellement le Québec et l'Ontario.

Toujours selon l'étude de la CCT, le Canada a reçu 427000 Allemands en 1995 , dont les deux tiers voyageaient dans un but d'agrément. Elle évalue que $16 \%$ (soit 68000 personnes $^{5}$ ) de ces touristes visitaient le Québec, alors que de tous les Français en visite au Canada, $83 \%$ venaient au Québec. La durée de voyage des touristes allemands était de 12 jours en moyenne, soit un peu moins que leurs congénères français. De son côté, le Québec recevait plus de 360000 visiteurs français en $1995^{9}$ (soit $83 \%$ des visiteurs du Canada), dont la moitié effectuaient leurs propres arrangements de voyages (vol seulement). Pour l'autre moitié, environ $60 \%$ arrivaient en circuit organisé et $40 \%$ avaient acheté la portion aérienne en plus d'une autre prestation (auto, hôtel, miniexcursion).
Cette même étude souligne que les voyagistes français et allemands suggèrent de mettre l'accent sur le style de vie traditionnelle autochtone, sur la culture et sur des activités d'aventure-nature dans une même programmation. Ils perçoivent le produit autochtone comme étant une composante unique de valeur ajoutée. Concrètement, le produit autochtone devrait pouvoir s' insérer facilement dans leurs circuits si l'on propose des prestations d'une demi à une journée ; il en va de même pour leurs forfaits spécialisés dans lesquels on pourrait insérer des expériences en mode de vie traditionnelle pouvant aller jusqu'à trois jours. L'intérêt possible des consommateurs allemands pour le produit autochtone est croissant, mais représente un potentiel de marché limité pour ce type de produit, alors que les consommateurs français confirment cet intérêt par une demande explicite auprès des voyagistes et des organismes de promotion.

\section{TOURISME AUTOCHTONE, TOURISME D'AVENTURE ET ÉCOTOURISME : TROIS VOLETS COMPLÉMENTAIRES}

Dans son étude sur le tourisme d'aventure au Canada ${ }^{10}$, la CCT (anciennement Tourisme Canada) souligne que a Le tourisme d'aventure est le secteur qui, dans l'industrie du tourisme en Amérique du Nord, connaît actuellement la plus grande expansion $»$. Donc, bon nombre de visiteurs sont intéressés par l'aventure. Ils représentent principalement $57 \%$ pour le Canada, 23,1 \% pour les États-Unis, $8,4 \%$ pour $1^{\prime}$ Allemagne, $4,4 \%$ pour la France, $2,5 \%$ pour le Royaume-Uni et $2 \%$ pour le Japon. Ces chiffres valent cependant pour l'ensemble du Canada et s'adressent à tous les types de tourisme d'aventure. Ce sont les producteurs d'aventure ontariens qui reçoivent le plus grand nombre de ces touristes avec plus de $22 \%$. alors que ceux du Québec en reçoivent moins de $6 \%$. Un rapport sur le tourisme d'aventure et l'écotourisme" donne un profil de ce segment de clientèle :

L'amateur typique était un homme $(54,4$ \%) à quelques exceptions près selon l'activité, et plus de la moitie des amateurs avaient entre 20 et 44 ans $(52,7 \%)$. Selon une étude 
réalisée en 1994. I'écotourisme atrirail autan d'hommes que de femmes. La plupart des écotouristes avaient entre 24 et 54 ans et une grande majorite d'entre eux $(82 \%)$ détenaient un diplôme d'études collegiales. I... I le Canada ne représente qu'une fraction du marché nord-américain du tourisme d'aventure et de l'écotourisme. De plus, selon une étude effectuée en 1993. $41 \%$ de la capacité de ce secteur est demeurée inutilisée.

En matière d'écotourisme, l'Ecotourism Society Fact Sheet Collecrion suggère quelques données sur les clientèles touristiques dans le monde :

Les ecolouristes sont des personnes âgées entre 35 et 54 ans (autant d'hommes que de femmes) : ils sont éduqués et scolarisés ; ils sont informés, lisent abondamment et ont beaucoup voyagé ; ils sont prêts à dépenser pour des voyages leur permettant de se retrouver au caur de la nature, de vive des expériences differentes, et de participer à des activités variées (observation d'animaux, échanges culturels, autres styles de vie, etc.).
Comme on le constate, la soucoupe est grande en matière de croisement de marchés. Il y aura maintenant lieu d'observer comment se positionnera le tourisme autochtone parmi toutes les activités d'aventure douce, de nature et de culture, intégrées à tout itinéraire du voyageur des années 2000 .
Marie-Andrée Delisle est présidente de Marie-Andrée Delisle et associés, une firme de services conseil et de formation spécifique au tourisme. Elle collabore étroitement avec diverses nations autochtones du Québec à des mandais de recherche et de développement touristique.

\section{LEXIQUE}

Autochtone : selon la définition du dictionnaire et aussi selon l'usage qu'en font les Français, un autochtone est une personne originaire du pays où elle habite. On dit aussi que les Autochtones du Canada sont les Amérindiens, terme que les Français utilisent en parlant des Indiens d'Amérique. Dans le langage courant québécois, on utilise le mot Autochtone pour identifier les premières nations qui ont habité le Canada.

Allochtone : personne qui n'est pas d'origine autochtone.

Aborigène : terme utilisé surtout pour les Premières Nations d'Australie.

Premières Nations : premiers peuples ayant habité le territoire canadien.

Communauté vs réserve : On utilise le mot communauté pour signifier le regroupement d'une nation en un lieu donné, plutôt que le mot réserve, dont le sens est devenu péjoratif.

\section{NOTES}

1 Prestation : produit qui n'est pas un bien matériel, mais qui satisfait l'usage et la consommation d'un individu (transport. hébergement, etc.). (Dupont, 1997 : 94)

2 Raid de motoneige regroupant des participants européens et québécois allochtones et autochtones, qui a eu lieu à l'hiver 19901991.

3 Organisme qui regroupe plusieurs entreprises touristiques montagnaises et atikamekw. dont la mission est de faire la promotion des entreprises, de les aider à se développer et à se former, et de créer de l'activité économique pour les communautés qu'il représente.

4 (Les Éditions La Griffe de l'Aigle, 1996)

5 (Les Editions Sylvain Harvey, 1997)

6 (Tourisme Québec, 1998 : 19)

7 (Williams et Richter, 1996)

8 Selon Le tourisme au Quebec en 1995. document de statistiques touristiques publié par Tourisme Québec en 1997, le Québec indiquait des données préliminaires en terme de visites-province pour 1996: 392600 visiteurs français, soit une augmentation de $7,2 \%$ sur 1995 et 115600 visiteurs Allemands, soit une augmentation de $47,5 \%$ sur 1995.

9 Le Québec recevait 393000 Français en 1996, alors que les chiffres pour 1997 sont estimés avec une baisse de $7 \mathrm{c}$.

10 (Tourisme Canada, 1995)

11 (Commission canadienne du tourisme, 1997 : 3, 5)

12 (The Ecotourism Society)

\section{BIBLIOGRAPHIE}

Dupont, Charles (1977), Vocabulaire du Tourisme, Linguatech, Montréal.

Les Éditions La Griffe de l'Aigle (1996), Le Quebec autochtone, Québec, $288 \mathrm{p}$.

Les Êditions Sylvain Harvey (1997), Le Québec amérindien et inuit, Collection Histoire de Voir, Québec, 58 pages.

Tourisme Québec, Politique de développement touristique (1998), Pour donner au monde le goüt du Québec, p. 19.

Williams, Peter W., et Richter, Christine (1996), European Tour Operator Distribution Channels for Aboriginal Tourism Products: France, Germany and Italy, Simon Fraser University. B.C., The Canadian Tourism Commission.

Tourisme Canada (1995), Le tourisme d'aventure au Canada - Aperçu du produit, du marché et du potentiel commencial - Direction du Canada.

Commission canadienne du tourisme (1997), Le tourisme d'aventure et l'écolourisme : Les enjeur.

The Ecotourism Society, The Ecotourism Sociery Fact Sheet Collection. North Bennington, VT.

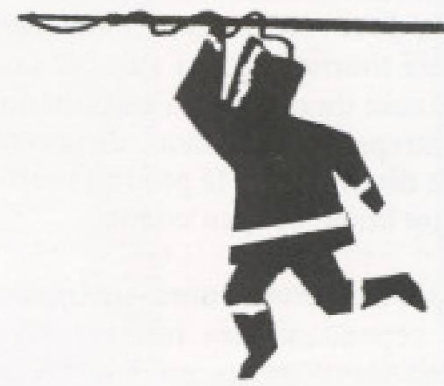

